\title{
Determinações e Relações dos Parâmetros Anaeróbios do RAST, do Limiar Anaeróbio e da Resposta Lactacidemica Obtida no Início, no Intervalo e ao Final de uma Partida Oficial de Handebol
}

\author{
Determinations and Relationships of the RAST Anaerobic Parameters, \\ Anaerobic Threshold and Lactacidemia Response Obtained at the \\ Beginning, Interval and the End of an Official Handball Match
}

Alex Zelanti Roseguini' Adelino Sanchez Ramos da Silva' Claudio Alexandre Gobatto'

1. Laboratório de Fisiologia Aplicada ao Esporte - Universidade Estadual Paulista "Júlio de Mesquita Filho", Rio Claro, São Paulo.

Endereço para correspondência: Adelino Sanchez Ramos da Silva, Avenida Miguel Damha, 1000 Residencial Damha I, unidade 214, Jardim Guanabara, CEP 13565-814, São Carlos, São Paulo. E-mail: adelinosanchez@hotmail.com

Submetido em 30/10/2006 Versão final recebida em 30/01/2007 Aceito em 18/10/2007

\begin{abstract}
RESUMO
O principal objetivo do presente estudo foi determinar os parâmetros anaeróbios obtidos através do RAST (Running-based Anaerobic Sprint Test) e o limiar anaeróbio de 12 atletas filiados a Federação Paulista de Handebol. Além disso, também procuramos verificar as correlações entre as variáveis do RAST, o limiar anaeróbio e a resposta lactacidemica obtida no início, no intervalo e ao final de uma partida oficial de handebol. As avaliações foram conduzidas em 2 dias. No primeiro dia, os atletas foram submetidos ao RAST e em seguida foi determinado o limiar anaeróbio de cada atleta através de um protocolo adaptado ao de Tegtbur et al. (1993). No segundo dia, durante a disputa de uma partida oficial de handebol, foram coletadas amostras de sangue para determinação das concentrações de lactato no início, no intervalo e ao final do jogo. Foi utilizado o teste Anova para dados repetidos, seguido pelo post hoc de Newman-Keuls quando necessário, com o intuito de comparar as concentrações de lactato obtidas ao longo da partida de handebol e a correspondente ao limiar anaeróbio. A análise de correlação de Pearson foi utilizada para verificar as relações entre os parâmetros anaeróbios, o limiar anaeróbio e as concentrações de lactato obtidas durante uma partida oficial de handebol. Para todos os casos o nível de significância foi pré-fixado em 5\%. Não foram verificadas correlações dos parâmetros anaeróbios do RAST e do limiar anaeróbio com as respostas lactacidemicas durante a partida de handebol. De acordo com os resultados obtidos no presente estudo, podemos concluir que embora o protocolo proposto para a avaliação do limiar anaeróbio também forneça parâmetros anaeróbios e se aproxime das situações reais do jogo de handebol, não foram encontradas correlações significativas entre as variáveis determinadas na avaliação com as concentrações de lactato obtidas durante a partida oficial de handebol.
\end{abstract}

Palavras-chave: parâmetros anaeróbios, capacidade aeróbia, lactacidemia

\section{ABSTRACT}

The main purpose of the present study was to determine the anaerobic parameters obtained by the RAST (Running-based Anaerobic Sprint Test) and the anaerobic threshold of twelve handballers affiliated with the Handball Federation of São Paulo. Moreover, we aimed to study the relationship of the RAST variables, the anaerobic threshold and the lactacidemia response obtained at the beginning, at the interval and at the end of a an official handball match. Measurements were carried out in two days. On the first day, the athletes performed the RAST and then, the anaerobic threshold was obtained by a protocol adapted from Tegtbur et al. (1993). On the second day, the athletes had blood samples collected to determine the blood lactate responses during the three different times of the handball game. Repeated measurements Anova test followed by post hoc Newman-Keuls test whenever needed, were used to compare the blood lactate concentrations during the handball match as well as that one corresponding to the anaerobic threshold. Pearson product-moment coefficient analysis was used to verify the relationships of the RAST variables, the anaerobic threshold and the blood lactate obtained at the beginning, in the middle and at the end of an official handball match. A significance level of $5 \%$ was chosen for all cases. Correlations between the RAST parameters and the anaerobic threshold with the blood lactate responses during the handball match have not been observed. According to our results, it is possible to conclude that the variables obtained by the protocol proposed to determine the anaerobic threshold did not present significant correlations with the blood lactate concentrations obtained during the official handball match.

Keywords: anaerobic parameters, aerobic capacity, lactatemia 


\section{INTRODUÇÃO}

Inicialmente, o jogo de handebol era disputado com a participação de 11 jogadores em um campo semelhante ao de futebol. Nos moldes atuais, o esporte é praticado em quadra $(40 \times 20 \mathrm{~m}$ ) e as equipes são constituídas por 7 atletas ( 6 de linha e 1 goleiro) que iniciam o jogo e mais 5 suplentes que podem participar da partida a qualquer momento, além disso, não há limite para as substituições ${ }^{(1)}$.

A duração total de uma partida de handebol é de 70 minutos, sendo 2 tempos de 30 min com 10 min de intervalo. A partida é disputada sem interrupção do tempo e os intervalos ocorrem apenas nas situações em que a arbitragem julgar necessário (Time out) ${ }^{(1)}$.

O handebol pode ser definido como uma modalidade esportiva coletiva caracterizada por atividades motoras completas ${ }^{(2)}$ que envolvem uma série de esforços, associados ou não com a manipulação da bola(3), de alta intensidade e curta duração com intervalos ativos ${ }^{(4)}$.

Principalmente devido ao tempo de jogo (60 min), o handebol pode ser caracterizado como uma modalidade esportiva em que grande parte do gasto energético dos atletas é suprida pelo metabolismo aeróbio(4). Além disso, Bergamasco et al. ${ }^{(5)}$ consideram a potência aeróbia como fator determinante para que os atletas apresentem uma recuperação adequada nos intervalos ativos que comumente ocorrem em uma partida de handebol. Por outro lado, a maioria das ações utilizadas pelos handebolistas para decidir uma partida (correr, saltar, driblar, fintar e arremessar) parece apresentar grande participação anaeróbia.

Embora exista uma vasta literatura internacional que objetivou contemplar diversos aspectos do handebol como força máxima ${ }^{(6)}$ e explosiva ${ }^{(6,7)}$, consumo máximo de oxigênio( ${ }^{(8,9)}$, e capacidade aeróbia ${ }^{(6,7,9)}$, em nível nacional, muito pouco tem sido realizado para caracterização fisiológica dos atletas dessa modalidade esportiva.

Dessa maneira, o principal objetivo do presente estudo foi determinar os parâmetros anaeróbios obtidos através do RAST (Runningbased Anaerobic Sprint Test) e o limiar anaeróbio de 12 atletas filiados a Federação Paulista de Handebol. Além disso, também procuramos verificar as correlações entre as variáveis do RAST, o limiar anaeróbio e a resposta lactacidemica obtida no início, no intervalo e ao final de uma partida oficial de handebol disputada nos Jogos Regionais de Sertãozinho em 2006.

\section{MATERIAIS E MÉTODOS}

\section{Participantes}

Participaram do estudo 12 jogadores de handebol do sexo masculino, pertencentes a uma equipe filiada a Federação Paulista de Handebol (FPH), que disputaram os Jogos Regionais de Sertãozinho em 2006. Os atletas e a comissão técnica foram previamente informados em relação aos procedimentos a que seriam submetidos e assinaram um termo de consentimento livre e esclarecido, aprovado pelo comitê de ética em pesquisa do Instituto de Biociências da Universidade Estadual Paulista "Júlio de Mesquita Filho" Campus de Rio Claro, autorizando a participação no estudo. A idade e as características biométricas dos participantes estão descritas na tabela 1.

Tabela 1. Idade e características biométricas dos 12 jogadores de handebol.

\begin{tabular}{c|c|c|c}
\hline Idade (anos) & MC (kg) & Estatura (m) & IMC $\left(\mathbf{k g} / \mathbf{m}^{2}\right)$ \\
\hline $19,33 \pm 1,15$ & $78,60 \pm 10,55$ & $1,80 \pm 0,07$ & $24,16 \pm 2,16$ \\
\hline $18-21$ & $64,10-98,70$ & $1,70-1,93$ & $20,31-28,83$ \\
\hline
\end{tabular}

Valores expressos em média \pm desvio padrão e valor mínimo - valor máximo. MC: massa corporal total.

IMC: índice de massa corporal.

\section{Desenho experimental}

As avaliações dos parâmetros anaeróbios determinados através do RAST e do limiar anaeróbio foram realizadas em uma quadra com piso de madeira, coberta e com dimensões oficiais (40 × 20 m) para a prática do handebol. A resposta lactacidemica foi obtida durante uma partida oficial de handebol disputada nos Jogos Regionais de Sertãozinho em 2006.

As análises das amostras sanguíneas foram realizadas no Laboratório de Fisiologia Aplicada ao Esporte (LAFAE) do Departamento de Educação Física da Universidade Estadual Paulista "Júlio de Mesquita Filho" campus de Rio Claro.

As avaliações foram conduzidas em dois dias. No primeiro dia, os atletas foram submetidos ao RAST e em seguida foi determinado o limiar anaeróbio de cada atleta através de um protocolo adaptado ao de Tegtbur et al. ${ }^{(10)}$. No segundo dia, durante a disputa de uma partida oficial de handebol válida pelos Jogos Regionais de Sertãozinho em 2006, foram coletadas amostras de sangue para determinação das concentrações de lactato no início, no intervalo e ao final do jogo.

\section{Parâmetros anaeróbios determinados através do RAST}

Os atletas foram submetidos a 6 esforços máximos de 35 metros, com 10 segundos de pausa passiva, e coleta de amostras de sangue para análise da lactacidemia no $3^{\circ}$, no $5^{\circ}$ e no $7^{\circ}$ minuto após o término dos esforços.

A potência $(\mathrm{P} ; \mathrm{W})$ para cada esforço foi obtida através do produto entre a massa corporal total do atleta $(M C ; \mathrm{kg})$ e a distância de cada esforço elevada ao quadrado $(35 \mathrm{~m}$ ). O resultado foi divido pelo tempo de cada esforço ( $T$; s) elevado ao cubo (Equação 1).

$$
\text { Equação } 1-P(W)=\frac{M C \times 35^{2}}{T^{3}}
$$

Foram registrados como parâmetros anaeróbios do RAST: a potência máxima (Pmáx; maior potência entre os 6 esforços), potência média (Pmédia; média entre as potências dos 6 esforços), potência mínima (Pmín; menor potência entre os 6 esforços), tempo total (TT; somatória do tempo dos 6 esforços), índice de fadiga (IF; W. s $^{-1}$; Equação 2) e as concentrações de lactato obtidas no $3^{\circ}$ ([Lac $]_{3 \min ;}$ mM), no $5^{\circ}\left([\mathrm{LaC}]_{5 \min }\right.$; $\mathrm{mM}$ ) e no $7^{\circ}$ minuto ([Lac $]_{7 \mathrm{~min}} ; \mathrm{mM}$ ) após a realização do protocolo.

$$
\text { Equação } 2 \text { - IF }\left(W \cdot s^{-1}\right)=\frac{(P m a x-P m i n)}{T T}
$$

Os resultados de Pmáx, Pmédia, Pmín e IF relativos à massa corporal total de cada atleta também foram utilizados como parâmetros anaeróbios do RAST.

\section{Limiar anaeróbio}

A intensidade de exercício correspondente ao limiar anaeróbio (iLan; $\mathrm{km} \cdot \mathrm{h}^{-1}$ ) foi determinada através de um protocolo adaptado ao modelo proposto originalmente por Tegtbur et al. ${ }^{(10)}$. O RAST foi utilizado como forma para indução à hiperlactacidemia. Posteriormente, os atletas permaneceram em repouso passivo durante 8 min, e a seguir iniciaram um protocolo incremental composto de 5 esforços submáximos realizados em vai e vem na distância de $35 \mathrm{~m}$. Os esforços tiveram duração de 3 min e foram realizados nas intensidades de 6,3; 8,4; 11,6; 13,6 e $14,7 \mathrm{~km} \cdot \mathrm{h}^{-1}$.

A intensidade de cada esforço foi controlada por estímulo sonoro (metrônomo) e ao final de cada estágio houve um intervalo de aproximadamente 45 s para coleta de amostras de sangue para posterior determinação das concentrações de lactato sanguíneo. A iLan foi considerada como a velocidade de corrida referente à menor concentração de lactato sanguíneo obtida pela derivada zero da equação polinomial de grau dois da curva lactacidemia versus velocidade de corrida. 


\section{Lactacidemia durante a partida de handebol}

Foram coletadas amostras de sangue para posterior determinação das concentrações de lactato no início ([Lac $]_{\text {iníicio }} \mathrm{mM}$ ), no intervalo ([Lac $]_{\text {intervalo; }} \mathrm{mM}$ ) e ao final ([Lac $]_{\text {finali }}$ mM) de uma partida oficial de handebol disputada nos Jogos Regionais de Sertãozinho em 2006.

\section{Coleta e análise sanguínea}

Foram coletados $25 \mu$ l de sangue arterializado do lóbulo da orelha através de capilares de vidro heparinizados e calibrados. O sangue foi depositado em tubos de 1,5 $\mathrm{mL}$ para microcentrífugas, contendo 50ul de fluoreto de sódio (NaF - 1\%), para posterior determinação da concentração de lactato sanguíneo (mM) em Lactímetro Eletroquímico Yellow Spring Instruments (YSI), modelo 1500 Sport.

\section{Análise estatística}

De acordo com o Shapiro Wilk's W test, o conjunto de dados apresentou distribuição normal e a homogeneidade foi verificada através do Levene's test. Dessa maneira, foi utilizado o teste Anova para dados repetidos, seguido pelo post hoc de Newman-Keuls quando necessário, com o intuito de comparar as concentrações de lactato obtidas no início, no intervalo, ao final da partida de handebol e a referente ao limiar anaeróbio. Além disso, foi utilizada a análise de correlação de Pearson para verificar as relações entre os parâmetros anaeróbios, o limiar anaeróbio e as concentrações de lactato obtidas durante uma partida oficial de handebol. Para todos os casos o nível de significância foi pré-fixado em $5 \%$.

\section{RESULTADOS}

A tabela 2 mostra os valores absolutos e relativos à massa corporal da potência máxima, potência média, potência mínima e índice de fadiga de 12 jogadores de handebol, obtidos pelo RAST.

Tabela 2. Valores absolutos e relativos à massa corporal da potência máxima (Pmáx), potência média (Pmédia), potência mínima (Pmín) e índice de fadiga (IF) de 12 jogadores de handebol.

\begin{tabular}{c|c|c|c}
\hline \multicolumn{4}{c}{ VALORES ABSOLUTOS } \\
\hline Pmáx (W) & Pmédia (W) & Pmín (W) & IF (W.s $\left.{ }^{-1}\right)$ \\
\hline $649,98 \pm 82,70$ & $529,32 \pm 69,71$ & $432,18 \pm 71,50$ & $5,84 \pm 1,99$ \\
\hline $465,14-748,14$ & $415,12-666,88$ & $331,74-568,57$ & $2,98-9,92$ \\
\hline \multicolumn{4}{c}{ VALORES RELATIVOS } \\
\hline Pmáx (W.kg-1) & Pmédia (W.kg ${ }^{-1}$ ) & Pmín (W.kg-1) & IF (W.kg-1.. $\mathbf{s}^{-1}$ ) \\
\hline $7,68 \pm 1,53$ & $6,26 \pm 1,26$ & $5,13 \pm 1,26$ & $0,42 \pm 0,11$ \\
\hline $5,70-10,03$ & $4,05-8,75$ & $3,24-7,46$ & $0,28-0,63$ \\
\hline
\end{tabular}

Valores expressos em média \pm desvio padrão e valor mínimo - valor máximo.

A tabela 3 mostra os valores da somatória do tempo dos 6 esforços, concentrações de lactato obtidas no $3^{\circ}$, no $5^{\circ}$ e no $7^{\circ}$ minuto após a realização do RAST, e concentração de lactato pico obtida nesse protocolo.

Na tabela 4 é possível visualizar os valores da intensidade de exercício e concentração de lactato sanguíneo referentes ao limiar anaeróbio determinado pelo protocolo adaptado ao de Tegtbur et al.(10), e valor do ajuste da equação polinomial de grau dois da curva lactacidemia versus velocidade de corrida.
Tabela 3. Valores da somatória do tempo dos 6 esforços (TT), concentrações de lactato obtidas no $3^{\circ}\left([\mathrm{LaC}]_{3 \min }\right)$, no $5^{\circ}\left([\mathrm{Lac}]_{5 \min }\right)$ e no $7^{\circ}$ minuto ([Lac $\left.]_{7 \min }\right)$ após a realização do RAST, e concentração de lactato pico ([Lac $]_{\text {pico }}$ ) obtida nesse protocolo.

\begin{tabular}{c|c|c|c|c}
\hline TT $(\mathbf{s})$ & {$[\mathrm{Lac}]_{3 \min }(\mathbf{m M})$} & {$[\mathrm{Lac}]_{5 \min }(\mathbf{m M})$} & {$[\mathrm{Lac}]_{7_{\text {min }}}(\mathbf{m M})$} & {$[\mathrm{Lac}]_{\text {pico }}(\mathbf{m M})$} \\
\hline $34,04 \pm 1,56$ & $7,52 \pm 1,70$ & $9,39 \pm 1,75$ & $9,34 \pm 1,88$ & $9,81 \pm 1,71$ \\
\hline $31,32-36,88$ & $5,22-10,35$ & $5,94-12,27$ & $6,66-12,00$ & $6,66-12,27$ \\
\hline
\end{tabular}

Valores expressos em média \pm desvio padrão e valor mínimo - valor máximo.

Tabela 4. Valores da intensidade de exercício (iLan) e concentração de lactato sanguíneo ([Lac]-iLan) referentes ao limiar anaeróbio determinado pelo protocolo adaptado ao de Tegtbur et al.(10), e valor do ajuste da equação polinomial de grau dois da curva lactacidemia versus velocidade de corrida.

\begin{tabular}{c|c|c}
\hline iLan $\left(\mathbf{k m} \cdot \mathbf{h}^{-1}\right)$ & {$[$ Lac] - iLan $(\mathbf{m M})$} & $\mathbf{R}^{\mathbf{2}}$ \\
\hline $11,04 \pm 0,83$ & $4,64 \pm 1,50$ & $0,91 \pm 0,08$ \\
\hline $9,93-12,84$ & $2,78-7,19$ & $0,76-0,99$ \\
\hline
\end{tabular}

Valores expressos em média \pm desvio padrão e valor mínimo - valor máximo.

De acordo com a tabela 5 é possível verificar que houve correlação significativa da potência máxima com a potência média; com a potência máxima e média relativas à massa corporal total e com a somatória do tempo dos 6 esforços do RAST. A potência média apresentou correlação significativa com a potência mínima; com a potência máxima, média e mínima relativas à massa corporal total; com a somatória do tempo dos 6 esforços do RAST e com a intensidade de exercício referente ao limiar anaeróbio.

Além disso, a potência mínima foi significativamente correlacionada com a potência máxima, média e mínima relativas à massa corporal total; com a somatória do tempo dos 6 esforços do RAST e com a intensidade de exercício referente ao limiar anaeróbio. A somatória do tempo dos 6 esforços do RAST apresentou correlação significativa com a intensidade de exercício referente ao limiar anaeróbio.

Tabela 5. Correlações significativas dos valores absolutos e relativos de potência máxima (Pmáx), potência média (Pmédia), potência mínima (Pmín), somatória do tempo dos 6 esforços (TT) e intensidade de exercício (iLan) referente ao limiar anaeróbio determinado pelo protocolo adaptado ao de Tegtbur et al.(10) de 12 jogadores de handebol.

\begin{tabular}{c|c|c|c|c|c}
\hline & Pmáx (W) & Pmédia (W) & Pmín (W) & TT (s) & iLan $\left(\mathbf{k m} \cdot \mathbf{h}^{-1}\right)$ \\
\hline Pmáx (W) & $x$ & $r=0,77^{*}$ & $x$ & $r=-0,74^{*}$ & $x$ \\
\hline Pmédia (W) & $r=0,77^{*}$ & $x$ & $r=0,89^{*}$ & $r=-0,99^{*}$ & $r=0,63^{*}$ \\
\hline Pmín (W) & $x$ & $r=0,89^{*}$ & $x$ & $r=-0,91^{*}$ & $r=0,65^{*}$ \\
\hline Pmáx (W.kg-1) & $r=0,74^{*}$ & $r=0,62^{*}$ & $r=0,62^{*}$ & $r=-0,63^{*}$ & $x$ \\
\hline Pmédia $\left({\left.\mathrm{W} . \mathrm{kg}^{-1}\right)}\right.$ & $r=0,59^{*}$ & $r=0,78^{*}$ & $r=0,84^{*}$ & $r=-0,79^{*}$ & $r=0,62^{*}$ \\
\hline Pmín (W.kg-1) & $x$ & $r=0,71^{*}$ & $r=0,88^{*}$ & $r=-0,73^{*}$ & $r=0,60^{*}$ \\
\hline TT (s) & $r=-0,74^{*}$ & $r=-0,99^{*}$ & $r=-0,91^{*}$ & $x$ & $r=-0,60^{*}$ \\
\hline
\end{tabular}

${ }^{*} p<0,05$

Na tabela 6 foi observada correlação significativa da concentração de lactato obtida no $5^{\circ}$ min após o término do RAST com a concentração de lactato obtida no 70 min, com a concentração pico de lactato sanguíneo e com a concentração de lactato referente ao limiar anaeróbio. 
A concentração de lactato obtida no $7^{\circ}$ min após o término do RAST foi significativamente correlacionada com a concentração pico de lactato sanguíneo e com a concentração de lactato referente ao limiar anaeróbio. Além disso, a concentração pico de lactato sanguíneo apresentou correlação significativa com a concentração de lactato referente ao limiar anaeróbio.

Tabela 6. Valores de correlação significativa das concentrações de lactato obtidas no $3^{\circ}\left([\mathrm{LaC}]_{3 \min }\right), 5^{\circ}\left([\mathrm{Lac}]_{5 \mathrm{~min}}\right)$ e $7^{\circ}$ minuto $\left([\mathrm{Lac}]_{7 \mathrm{~min}}\right.$ ) após a realização do RAST, da concentração de lactato pico obtida nesse protocolo ([Lac $]_{\text {pico }}$ ) e da concentração de lactato referente ao limiar anaeróbio determinado pelo protocolo de lactato mínimo de 12 jogadores de handebol.

\begin{tabular}{c|c|c|c|c}
\hline & {$\left[\mathrm{Lac}_{3 \min }(\mathrm{mM})\right.$} & {$\left[\mathrm{Lac}_{5 \text { min }}(\mathrm{mM})\right.$} & {$[\mathrm{Lac}]_{7_{\text {min }}}(\mathrm{mM})$} & {$[\mathrm{Lac}]_{\text {pico }}(\mathrm{mM})$} \\
\hline$[\mathrm{Lac}]_{3 \min }(\mathrm{mM})$ & $x$ & $x$ & $x$ & $x$ \\
\hline$[\mathrm{Lac}]_{5 \min }(\mathrm{mM})$ & $x$ & $x$ & $r=0,81^{*}$ & $r=0,94^{*}$ \\
\hline$[\mathrm{Lac}]_{7_{\min }}(\mathrm{mM})$ & $x$ & $r=0,81^{*}$ & $x$ & $r=0,90^{*}$ \\
\hline$[\mathrm{Lac}]_{\text {pico }}(\mathrm{mM})$ & $x$ & $r=0,94^{*}$ & $r=0,90^{*}$ & $x$ \\
\hline$[\mathrm{Lac}]-\mathrm{iLan}(\mathrm{mM})$ & $x$ & $r=0,76^{*}$ & $r=0,77^{*}$ & $r=0,76^{*}$ \\
\hline
\end{tabular}

${ }^{*} p<0,05$.

De acordo com a tabela 7, as concentrações de lactato referente ao limiar anaeróbio e as obtidas no intervalo e ao final da partida de handebol foram significativamente maiores do que no início do jogo. Além disso, a concentração de lactato ao final da partida foi estatisticamente inferior do que concentração de lactato referente ao limiar anaeróbio.

Tabela 7. Valores das concentrações de lactato correspondente ao limiar anaeróbio a as obtidas no início ([Lac $]_{\text {inicio }}$ ), no intervalo ([Lac $]_{\text {intervalo }}$ ), ao final ([Lac $\left.]_{\text {finali }} ; \mathrm{mM}\right)$ de uma partida oficial de handebol disputada por 8 atletas nos Jogos Regionais de Sertãozinho em 2006.

\begin{tabular}{c|c|c|c}
\hline$[\mathrm{Lac}]-\operatorname{Lan}(\mathrm{mM})$ & {$[\mathrm{Lac}]_{\text {início }}(\mathrm{mM})$} & {$[\mathrm{Lac}]_{\text {intervalo }}(\mathrm{mM})$} & {$[\mathrm{Lac}]_{\text {final }}(\mathrm{mM})$} \\
\hline $4,91 \pm 1,49^{*}$ & $1,02 \pm 0,26$ & $4,17 \pm 1,13^{*}$ & $3,14 \pm 1,51^{*} \dagger$ \\
\hline $3,26-7,19$ & $0,48-1,19$ & $2,91-5,73$ & $2,07-6,75$ \\
\hline
\end{tabular}

Valores expressos em média \pm desvio padrão e valor mínimo - valor máximo.

* Diferença significativa em relação a [Lac $]_{\text {início. }}$

† Diferença significativa em relação a [Lac] - Lan.

\section{DISCUSSÃO}

O RAST foi desenvolvido pela Universidade de Wolverhampton (Reino Unido) com o objetivo de determinar as potências máxima, média e mínima, além do índice de fadiga na corrida. Zacharogiannis et al.(11) verificaram altas correlações significativas entre as potências máxima e média determinadas pelo RAST e pelo teste de Wingate.

Além disso, os parâmetros determinados através do RAST têm apresentado correlações significativas com as performances máximas de 100, 200 e $400 \mathrm{~m}$ na corrida ${ }^{(12)}$.

Embora alguns autores ${ }^{(11-13)}$ tenham utilizado o RAST para mensuração das potências máxima, média, mínima e índice de fadiga, não foi encontrado na literatura nenhum trabalho que usou esse protocolo para a avaliação de atletas de handebol. Considerando o estudo de Zacharogiannis et al. ${ }^{(11)}$, é possível compararmos nossos resultados com os achados de Rannou et al. ${ }^{(14)}$ e Bencke et al. ${ }^{(15)}$.
Rannou et al. ${ }^{(14)}$ verificaram valores superiores de potência máxima absoluta, potência máxima relativa à massa corporal e concentração de lactato obtida após 5 min da realização do teste de Wingate para jogadores nacionais $\left(1067,0 \pm 34,0 \mathrm{~W} ; 14,5 \pm 0,5 \mathrm{~W} \mathrm{~kg}^{-1}\right.$; e 14,0 $\pm 0,6$ $\mathrm{mM})$ e internacionais $\left(1172,0 \pm 47,0 \mathrm{~W} ; 14,8 \pm 0,7 \mathrm{~W}^{\mathrm{kg}}{ }^{-1}\right.$; e 15,3 $\pm 0,7$ $\mathrm{mM}$ ) de handebol quando comparados aos resultados encontrados em nosso experimento $\left(649,98 \pm 82,70 \mathrm{~W} ; 7,68 \pm 1,53 \mathrm{~W} \cdot \mathrm{kg}^{-1}\right.$; e 9,39 $\pm 1,75 \mathrm{mM})$.

Essa discrepância verificada entre os resultados obtidos por Rannou et al. ${ }^{(14)}$ e pelo nosso estudo poderia ser explicada pelo nível de condicionamento físico dos atletas avaliados. Contudo, os valores de potência máxima absoluta $(851,0 \pm 34,0 \mathrm{~W})$, potência máxima relativa à massa corporal $\left(12,3 \pm 0,6 \mathrm{~W} \cdot \mathrm{kg}^{-1}\right)$ e concentração de lactato $(11,5$ $\pm 0,4 \mathrm{mM}$ ) obtida após 5 min da realização do teste de Wingate em indivíduos sedentários ${ }^{(14)}$ também foram superiores aos valores observados em nossos atletas.

Dessa maneira, contrariando os achados de Zacharogiannis et al. ${ }^{(11)}$, acreditamos que haja uma dependência da determinação dos parâmetros anaeróbios destacados anteriormente com relação ao protocolo utilizado para a avaliação. No estudo de Zacharogiannis et al.(11) a amostra foi composta por indivíduos ativos, portanto existe a necessidade de verificar se as correlações significativas encontradas entre as potências máxima e média determinadas pelo RAST e pelo teste de Wingate também ocorrem em atletas.

Bencke et al. ${ }^{(15)}$ verificaram valores inferiores de potência máxima (298 - 537 W) e média (268 - 477 W) em atletas de handebol quando comparados aos nossos achados (465,14 - 748,14 W; 415,12 - 666,88 W). No entanto, a principal diferença entre as amostras analisadas é a idade, visto que Bencke et al. ${ }^{(15)}$ estudaram handebolistas com 12,5 anos.

Nossos atletas apresentaram correlações significativas entre os valores absolutos e relativos à massa corporal de potência máxima ( $r$ $=0,74)$, média $(r=0,78)$ e mínima $(r=0,88)$. Esses resultados indicam que para o grupo avaliado, a massa corporal total não influenciou na performance anaeróbia.

Alguns estudos ${ }^{(6,7,9)}$ foram realizados com o intuito de determinar a capacidade aeróbia de atletas de handebol, no entanto, nenhum deles apresentou os resultados referentes ao limiar anaeróbio $\left(\mathrm{km} \cdot \mathrm{h}^{-1}\right)$. Delamarche et al. ${ }^{(9)}$ constataram em cicloergômetro que o consumo de oxigênio no OBLA (Onset of Blood Lactate Concetration) foi de 3,53 $\pm 0,22$ L. min $^{-1}$.

Gorostiaga et al. ${ }^{(6)}$ estudaram o efeito de 6 semanas de treinamento de resistência nas concentrações de lactato sanguíneo referentes as velocidades de 10 e $11 \mathrm{~km} \cdot \mathrm{h}^{-1}$ em handebolistas adolescentes (15,1 \pm 0,7 anos). A concentração média de lactato sanguíneo na intensidade de $11 \mathrm{~km} \cdot \mathrm{h}^{-1}$ foi de 4,0 \pm 1,8 mM na situação pré-treinamento. Em nosso estudo, verificamos que a iLan dos atletas foi de 11,04 $\pm 0,83 \mathrm{~km} \cdot \mathrm{h}^{-1} \mathrm{e}$ a [Lac] - iLan de 4,64 \pm 1,50 mM.

Gorostiaga et al. ${ }^{(7)}$ consideraram a intensidade de exercício correspondente a 3 mM como parâmetro de capacidade aeróbia. Os handebolistas (31 \pm 4 anos) apresentaram capacidade aeróbia de 11,9 0 0,9 $\mathrm{km} \cdot \mathrm{h}^{-1}$ no início do período preparatório básico de uma periodização com duração de 45 semanas.

A principal vantagem do método de determinação do limiar anaeróbio proposto no presente estudo é que a partir de apenas 1 avaliação podemos obter, além da capacidade aeróbia, uma série de parâmetros anaeróbios através do RAST. Além disso, o fato dos atletas realizarem a parte incremental do protocolo em corridas de vai e vem de $35 \mathrm{~m}$ torna a avaliação mais próxima das situações reais do jogo de handebol (transição ataque - defesa). 
Interessantemente os atletas avaliados apresentaram correlações significativas entre a iLan, os valores absolutos ( $r=0.63$ e $r=0.65)$ e relativos á massa corporal total $(r=0.62$ e $r=0.60)$ de potência média e mínima, e a somatória do tempo dos 6 esforços do RAST ( $r=-0.60)$. Esses resultados indicam que os atletas com a capacidade aeróbia mais desenvolvida também foram melhores em alguns aspectos da avaliação anaeróbia.

Existem basicamente duas hipóteses que poderiam explicar as correlações significativas destacadas acima. Na primeira, devido às características do jogo de handebol que envolve uma base aeróbia e ações anaeróbias, o treinamento específico da modalidade poderia conduzir a adaptações semelhantes dos sistemas energéticos.

A segunda hipótese leva em consideração o fato dos parâmetros anaeróbios e da capacidade aeróbia terem sido obtidos na mesma avaliação. Em outro estudo do nosso grupo ${ }^{(16)}$, não observamos correlações significativas entre a capacidade aeróbia e os parâmetros de performance anaeróbia alática determinados por avaliações distintas ao longo de uma periodização desenvolvida em futebolistas profissionais.

Em nosso estudo, todos os atletas avaliados apresentaram pico da concentração do lactato sanguíneo no intervalo da partida $(4,17 \pm 1,13$ mM) e esse valor não apresentou diferença significativa da [Lac]- Lan (4,91 \pm 1,49 mM) o que enfatiza a predominância aeróbia do esporte. Delamarche et al. ${ }^{(9)}$ constataram que a concentração pico de lactato sanguíneo durante uma partida de handebol foi de 6,73 \pm 1,81 mM e que esse valor esteve acima da [Lac] referente ao OBLA (4mM).
O fato de que em um jogo de handebol não existe limite para substituições pode favorecer a recuperação passiva dos atletas e explicar os baixos valores lactacidemicos encontrados ao longo da partida analisada. Especificamente nesse caso, a fragilidade do adversário (diferença de 10 gols ao final do jogo) pode ter influenciado no aspecto motivacional dos atletas que consequentemente podem ter diminuído o ritmo de jogo, o que provavelmente foi fundamental na queda de $24,70 \%$ da concentração de lactato mensurada no intervalo e ao final da partida.

De acordo com os resultados obtidos no presente estudo, podemos concluir que embora o protocolo proposto para a avaliação do limiar anaeróbio também forneça parâmetros anaeróbios e se aproxime das situações reais do jogo de handebol, não foram encontradas correlações significativas entre as variáveis determinadas na avaliação com as concentrações de lactato obtidas durante a partida oficial de handebol.

Além disso, mais estudos precisam ser realizados com esse protocolo de determinação do limiar anaeróbio para que se possam elucidar com maior clareza os mecanismos que expliquem as correlações significativas entre o limiar anaeróbio e os parâmetros obtidos pelo RAST.

\section{AGRADECIMENTO}

Apoio Financeiro: Fapesp (04/15241-4).

Todos os autores declararam não haver qualquer potencial conflito de interesses referente a este artigo.

\section{REFERÊNCIAS BIBLIOGRÁFICAS}

1. Confederação Brasileira de Handebol. Handebol Regras Oficiais 2006/2009. 2a ed. São Paulo: Phorte Editora, 2006.

2. Retechuki A, Silva SG. Resposta da freqüência cardíaca no jogo de handebol em escolares do sexo feminino. Revista Treinamento Desportivo 2001; 6: 38-43.

3. Eleno T, Barela JA, Kokubun E. Tipos de esforço e qualidades físicas do handebol. Rev Bras Cien Esporte 2002; 24: 83-98.

4. Souza J, Ribeiro, MA, Ramires, GA, Brevilhéri, JC. Evolução da potência aeróbia máxima em atletas de handebol adulto durante o período de preparação. Revista Treinamento Desportivo 2000; 5: 29-34.

5. Bergamasco JGP, Benchimol LF, Filho LFMA, Vargas P, Carvalho T, Moraes AM. Análise da freqüência cardíaca e do VO2max em atletas universitários de handebol através do teste do vai-e-vem 20 metros. Movimento \& Percepção 2005; 5: 146-62.

6. Gorostiaga EM, Izquierdo M, Iturrelde P, Ruesta M, Ibáñez J. Effects of heavy resistance training on maximal and explosive force production, endurance and serum hormones in adolescent handball players. Eur J Appl Physiol 1999; 80: 485-93.

7. Gorostiaga EM, Granados C, Ibáñez J, Gonzales-Badillo JJ, Izquierdo M. Effects of an entire season on physical fitness changes in elite male handball players. Med Sci Sports Exerc 2006; 38: 357-66.

8. Jensen J, Jacobsen ST, Hetland S, Tveit P. Effect of combined endurance, strength and sprint training on maximal oxygen uptake, isometric strength and sprint performance in female elite handball players during a season. Int J Sports Med 1997; 18: 354-58.
9. Delamarche P, Gratas A, Beillot J, Dassonville J, Rochcongar P, Lessard Y. Extent of lactic anaerobic metabolism in handballers. Int J Sports Med 1987; 8: 55-9.

10. Tegtbur U, Busse MW, Braumann KM. Estimation of an individual equilibrium between lactate production and catabolism during exercise. Med Sci Sports Exerc 1993; 25: 620-7.

11. Zacharogiannis E, Paradisis G, Tziortzis S. An evaluation of tests of anaerobic power and capacity. Med Sci Sports Exerc 2004; 36: S116.

12. Paradisis GP, Tziortzis S, Zacharogiannis E, Smirniotou A, Karatzanos L. Correlation of the running-based anaerobic sprint test (RAST) and performance on the $100 \mathrm{~m}, 200 \mathrm{~m}$ and $400 \mathrm{~m}$ distance tests. J Hum Mov Stud 2005; 49: 77-92.

13. Balčiūnas M, Stonkus S, Abrantes C, Sampaio J. Long term effects of different training modalities on power, speed, skill and anaerobic capacity in young male basketball players. J Sports Sci Med 2006; 5: 163-70.

14. Rannou F, Prioux J, Zouhal H, Gratas-Delamarche A, Delamarche P. Physiological profile of handball players. J Sports Med Phys Fitness 2001; 41:349-53.

15. Bencke J, Damsgaard R, Saekmose A, Jorgensen P, Jorgensen K, Klausen K. Anaerobic power and muscle strength characteristics of 11 years old elite and non-elite boys and girls from gymnastics, team handball, tennis and swimming. Scand J Med Sci Sports 2002; 12: 171-8.

16. Silva ASR, Santhiago V, Papoti M, Gobatto C. Comportamento das concentrações séricas e urinárias de creatinina e uréia ao longo de uma periodização desenvolvida em futebolistas profissionais: Relações com a taxa de filtração glomerular. Rev Bras Med Esporte 2006; 12: 327-32. 